\section{The Neurochip BCI: Towards a Neural Prosthesis for Upper Limb Function}

Andrew Jackson, Chet T. Moritz, Jaideep Mavoori, Timothy H. Lucas, and Eberhard E. Fetz

\begin{abstract}
The Neurochip BCI is an autonomously operating interface between an implanted computer chip and recording and stimulating electrodes in the nervous system. By converting neural activity recorded in one brain area into electrical stimuli delivered to another site, the Neurochip BCI could form the basis for a simple, direct neural prosthetic. In tests with normal, unrestrained monkeys, the Neurochip continuously recorded activity of single neurons in primary motor cortex for several weeks at a time. Cortical activity was correlated with simultaneously-recorded electromyogram (EMG) activity from arm muscles during free behavior. In separate experiments with anesthetized monkeys, we found that microstimulation of the cervical spinal cord evoked movements of the arm and hand, often involving multiple muscles synergies. These observations suggest that spinal microstimulation controlled by cortical neurons could help compensate for damaged corticospinal projections.
\end{abstract}

Index Terms-Brain-computer interface (BCI), motor cortex, neural prosthetics, spinal cord injury.

\section{INTRODUCTION}

Here, we describe work with a battery-powered, implanted computer chip that could potentially serve as a neural prosthetic to aid upper limb function following injury of the spinal cord. Regaining arm and hand function is considered the highest priority by quadriplegic patients [1] and accurate control of these movements depends on corticospinal projections originating largely in primary motor cortex (M1) [2]. Previous research has shown that the activity of cells recorded in M1 can be used to control computer cursors and robotic devices [3]-[5]. We are investigating the possibility of an artificial corticospinal connection using M1 activity to continuously control microstimulation delivered in the spinal cord, which could help to restore function to the patient's own limbs.

We report progress in three areas. First, we review the main features of our Neurochip BCI, an implanted brain-computer interface (BCI) developed for neural recordings and microstimulation in macaque monkeys. The macaque is a good model for human upper limb control since its corticospinal system resembles that of humans. The Neurochip BCI can operate autonomously for extended periods of time in completely unrestrained monkeys, enabling us to study the long-term effects of incorporating prosthetic connections into the nervous system. Next, we describe recent experiments in which the Neurochip BCI was used to study the relationship between motor cortex cell firing rate and muscle activity during extended periods of free behavior. We show that this system can obtain stable, movement-related neural activity from M1, a prerequisite for an eventual prosthetic. Finally, we summarize experiments demonstrating that low-intensity intraspinal

Manuscript received July 18, 2005; revised March 10, 2006; accepted March 20,2006 . This work was supported in part by the National Institutes of Health under Grant NS12542 and Grant RR00166, in part by the Office of Naval Research under Grant N00014-01-1-0676, in part by the University of Washington Royalty Research Fund, and in part by The David and Lucile Packard Foundation.

A. Jackson, C. T. Moritz, T. H. Lucas, and E. E. Fetz are with the Department of Physiology and Biophysics, University of Washington, Seattle, WA 98195 USA (e-mail: jacksona@u.washington.edu).

J. Mavoori is with the Department of Electrical Engineering, University of Washington, Seattle, WA 98195 USA.

Digital Object Identifier 10.1109/TNSRE.2006.875547

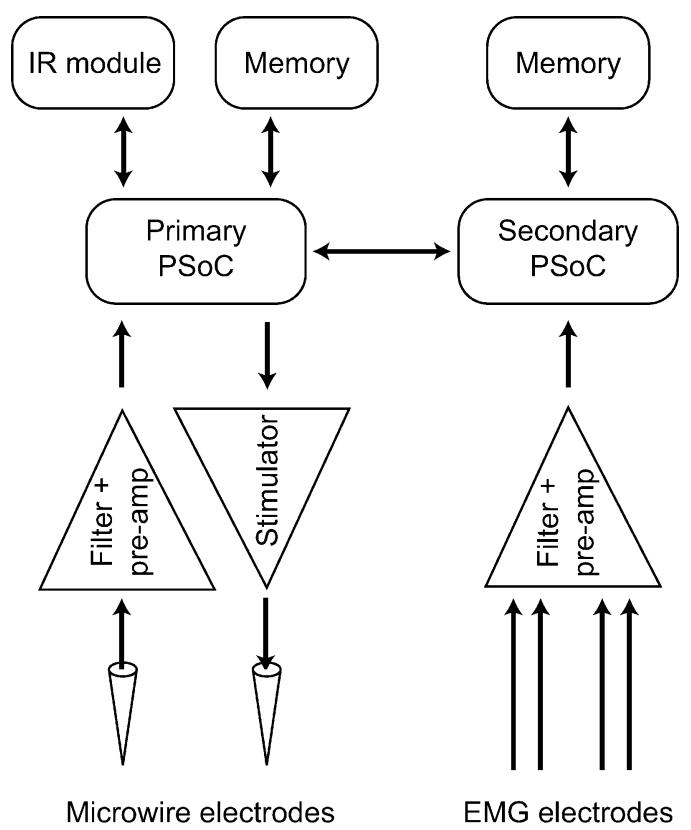

Fig. 1. Schematic of the Neurochip functional blocks. Parallel PSoC microcontrollers record neural and muscle signals to independent memory modules. Primary PSoC also controls a constant-current stimulator circuit and communicates via IR to a PC or hand-held PDA.

microstimulation can evoke movements of the arm and hand, typically involving multiple muscles.

\section{NEUROCHIP BCI}

The Neurochip BCI is an autonomous, battery-powered BCI. Using implantable electronics, we are able to collect data in unrestrained monkeys without the high power consumption and short battery life of radio-telemetry systems [6], [7]. Onboard spike processing and a stimulator circuit allow for real-time bidirectional interface with the nervous system. Here, we give a brief overview of the system and describe recent modifications that allow EMG activity to be recorded simultaneously with neural activity.

The electronic circuitry and battery are enclosed within a percutaneous titanium casing measuring $5.5 \mathrm{~cm} \times 5 \mathrm{~cm} \times 3 \mathrm{~cm}$ attached to the monkey's skull; the entire implant weighs $56 \mathrm{~g}$. A 2/3 AA-sized $3.3 \mathrm{~V}$ lithium battery powers the circuit for up to $40 \mathrm{~h}$, depending on the recording configuration. Neural data is acquired from one of 12 microwire electrodes $(50-\mu \mathrm{m}$-diameter teflon-insulated titanium, A-M Systems, Inc.) chronically implanted in primary motor cortex. Leads run subcutaneously from the head casing to a connector on the monkey's back. Two pairs of stainless-steel wires inserted percutaneously into forearm muscles can be attached to this connecter for recording electromyogram (EMG) signals.

Fig. 1 shows a schematic of the Neurochip architecture. At the heart of the electronics are two Programmable System-on-Chips (PSoCs) (Cypress Semiconductor Corporation) operating in parallel. The primary PSoC samples data from one of the cortical microwires at $11.7 \mathrm{ksps}$ and handles infrared (IR) communication. A secondary PSoC multiplexes and samples two differential, rectified EMG signals at $2 \mathrm{ksps}$ per channel. Inter-PSoC communication for synchronizing recordings and relaying data is handled by an asynchronous serial bus. Front-end signal processing includes band-pass filtering and amplification $(500 \mathrm{~Hz}-5 \mathrm{kHz}, 1500 \times$ with a further $1-48 \times$ variable gain for neural signals; $20 \mathrm{~Hz}-2 \mathrm{kHz}, 250 \times$ with further $1-48 \times$ followed by 
full-wave rectification for EMG). Each PSoC stores data to independent $8-\mathrm{Mb}$ flash memory chips. The Neurochip BCI also incorporates stimulation circuitry capable of delivering biphasic constant-current stimuli of up to $100 \mu \mathrm{A}$ to a different microwire electrode [7].

Each PSoC has an 8-bit microprocessor core, used to detect action potentials in the neural signal, calculate firing rate and EMG envelopes, store data and control stimulation. The spike discrimination algorithm consists of a threshold level which the signal must exceed, followed by two adjustable time-amplitude windows through which the signal must pass. Spike rate and average EMG level can be calculated and stored over a user-defined time-bin, resulting in considerable memory savings. The primary PSoC's $8 \mathrm{Mb}$ memory bank can hold $85 \mathrm{~s}$ of raw neural data sampled at $11.7 \mathrm{ksps}$ while the secondary PSoC's memory bank can hold $256 \mathrm{~s}$ of dual-channel raw EMGs sampled at $2 \mathrm{ksps}$. However, if spike rate is compiled for consecutive $100 \mathrm{~ms}$ bins along with simultaneous average EMGs, the Neurochip can store over $27 \mathrm{~h}$ of continuous data. Short sections of raw signal can be interspersed during the recording period, allowing discrimination quality to be confirmed throughout. The Neurochip operates autonomously after the recording, discrimination and stimulation parameters have been set via the IR link. Typically we download data via IR and replace the battery daily for continuous operation over many months. In one animal, this system has been recording data for over 16 months.

\section{NEURAL AND EMG RECORDINGS DURING NATURAL BEHAVIOR}

The relationship between motor cortical activity and movements has traditionally been studied in awake animals by recording neural spiking during the performance of repetitive, trained tasks under restrained conditions [2], [8], [9]. This approach offers technical and methodological advantages. Stable recordings can be obtained most easily with the head fixed, while mains-powered rack amplifiers and acquisition systems can be used to collect data. Furthermore, limiting the range and dimensionality of possible movements aids the interpretation of task-related cortical activity. By contrast, a neural prosthetic intended to restore a wide range of motor behavior would need to extract signals from cortical neurons across a wide range of behavior. Toward this end, we used our Neurochip system to investigate the relationship between neural and EMG activity during extended periods of free behavior in two monkeys.

Fig. 2(a) shows a neural signal sampled by the Neurochip from a microwire electrode in M1, and rectified EMG activity recorded simultaneously from a wrist extensor muscle. Fig. 2(b) shows 5 min of spike firing rate and mean EMG level during free behavior. This section was taken from a longer record covering $12 \mathrm{~h}$ of day and nighttime activity.

The relationship between cortical and EMG activity can be revealed by cross-correlation methods based on simple linear regression analysis. The plot in Fig. 2(c) was compiled from $6 \mathrm{~h}$ of continuous daytime firing rate and average rectified EMG activity, recorded over the same consecutive $100 \mathrm{~ms}$ bins. The regression coefficient $(r)$ was calculated between the aligned signals (zero-lag) and for the EMG shifted forwards or backwards in time relative to the firing rate by up to $5 \mathrm{~s}$. A positive correlation peak around zero-lag $(r=0.28)$ is seen between this cell and activity of the wrist extensor muscle (ECR), as well as a negative correlation trough with an antagonist wrist flexor (FCU). Most cells showed peak correlations with arm and extrinsic hand muscles of $0.1<r<0.4$, with cell firing leading muscle activity by $0-100 \mathrm{~ms}$, consistent with a causal role in generating motor commands. These correlation coefficients are slightly lower than those typically obtained during repetition of trained tasks in a restricted workspace, which are usually in the range of $0.2-0.6$ [9]. Nevertheless, for our proposed neural prosthetic, it is encouraging that robust correlations can
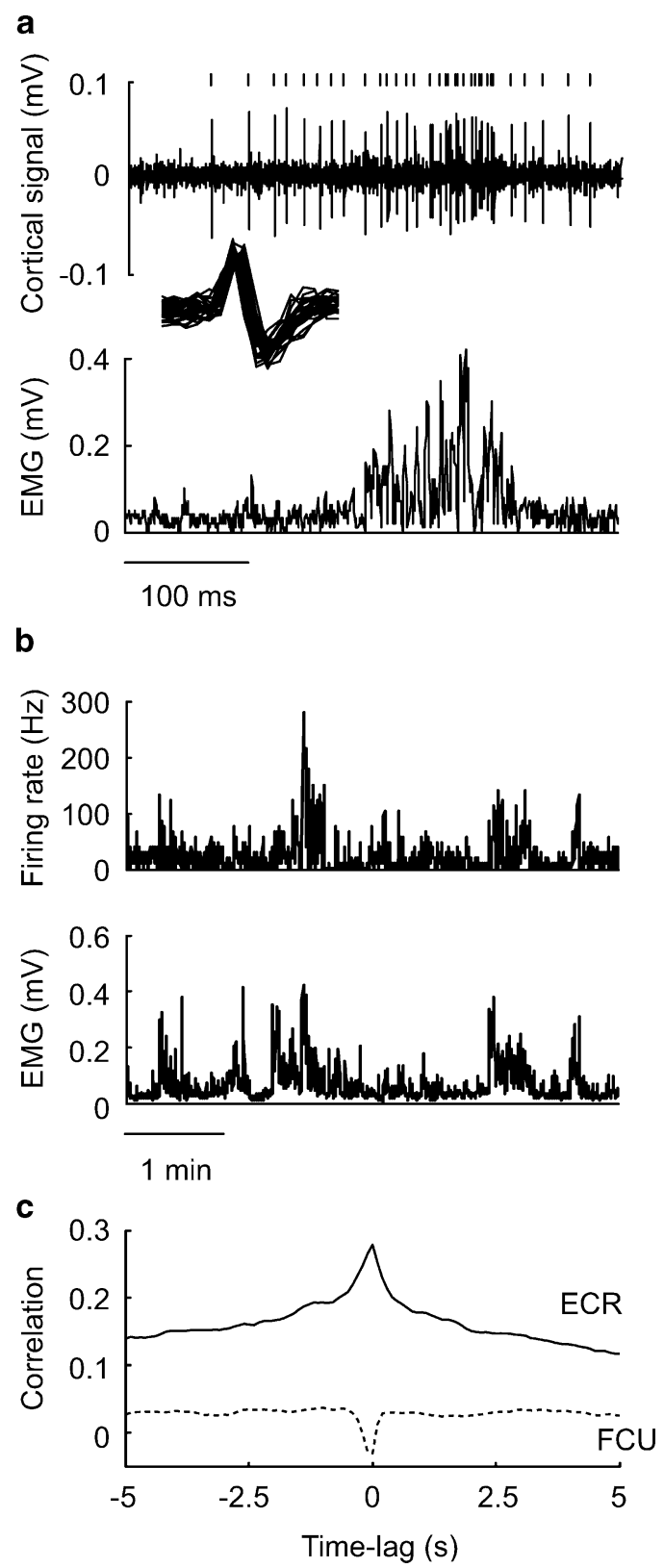

Fig. 2. (a) Raw recording from a microwire electrode in M1 and simultaneous rectified EMG from extensor carpi radialis muscle (ECR) recorded with the Neurochip. Discriminated action potentials are marked with ticks above, and the superimposed waveforms are inset. (b) Longer section of data recorded as mean firing rate and rectified EMG over consecutive $100 \mathrm{~ms}$ time-bins. (c) Cross correlation between spike firing rate and ECR activity over $6 \mathrm{~h}$ of unrestrained behavior. Also shown is the cross correlation with flexor carpi ulnaris muscle (FCU, dashed line).

be observed between single motor cortex neurons and individual arm muscles over long periods of completely unrestrained behavior.

Using the Neurochip BCI, we were able to continuously monitor the activity of single cells for periods of several weeks at a time. Correlations between cell firing and muscle activity remained consistent from day to day, although in some cases, the overall firing rate of a cell could drift slightly over extended periods of time.

\section{Microstimulation OF THE SPinAl CORD}

To study the output effects evoked by intraspinal microstimulation, we conducted mapping experiments in three sedated primates. These 

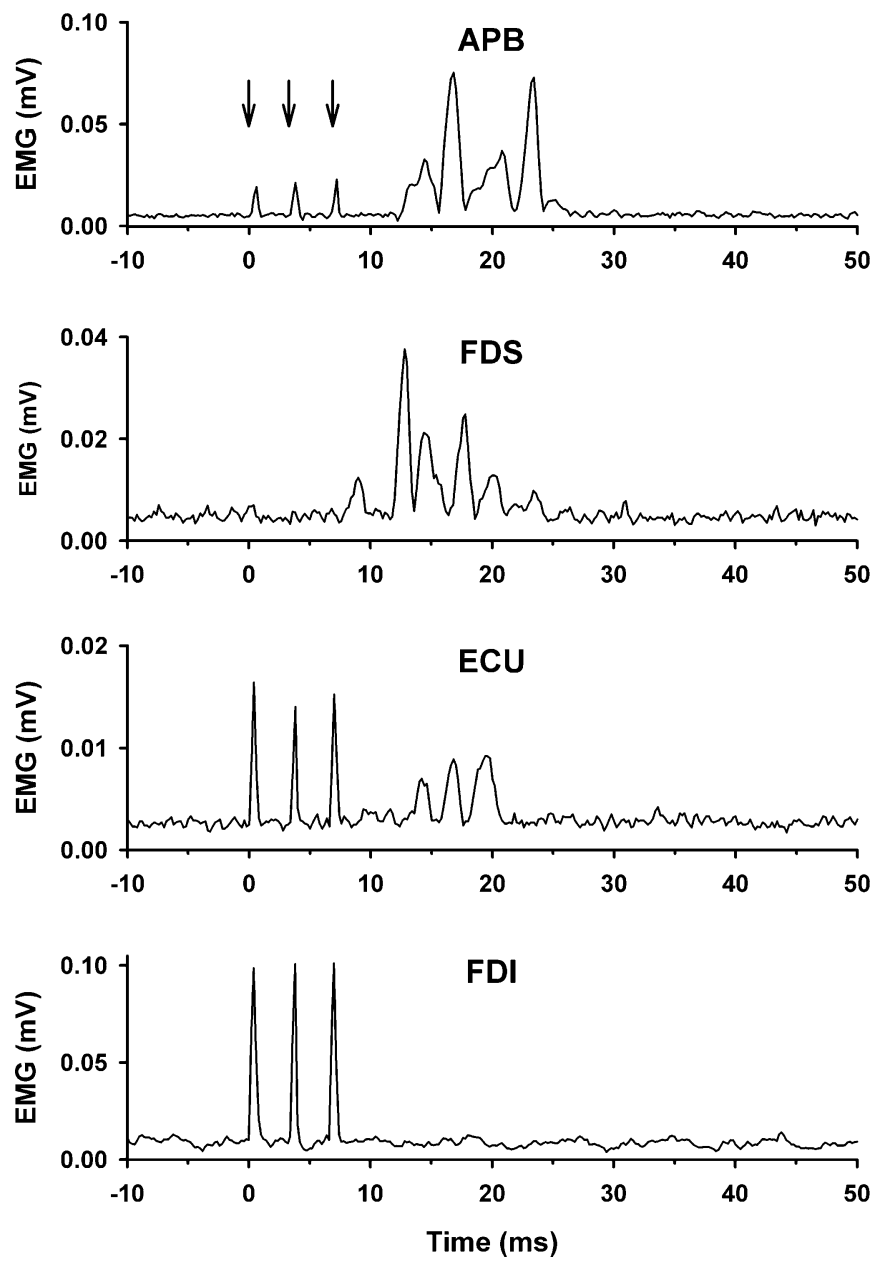

Fig. 3. Muscle activity evoked by microstimulation of lamina VII in the rostral C7 segment of the spinal cord. Train of three biphasic current pulses at $20 \mu \mathrm{A}$ (arrows) is just above threshold for eliciting a visible gripping movement of the monkey's hand. Multiple EMG responses in muscles controlling the wrist (ECU), fingers (FDS), and thumb (APB) are seen.

experiments will guide the placement of chronically indwelling stimulating electrodes [10] that may be controlled in awake animals by the Neurochip BCI.

During an initial surgery, a laminectomy over four cervical vertebrae was covered by a recording chamber attached by screws in the lateral masses [11]. In subsequent sessions with the monkeys lightly sedated, we delivered brief trains of biphasic, constant-current stimuli through tungsten microelectrodes advanced through successive sites in the cord, recording correlated EMG signals at the minimum current that elicited a visible movement. Low movement thresholds (10-90 $\mu \mathrm{A}$ ) within the range of our Neurochip stimulator [7] were obtained throughout most of the lower cervical spinal cord (76\% of stimulation sites). Fig. 3 shows responses to three stimuli of $20 \mu \mathrm{A}$ evoked in multiple hand and forearm muscles, associated with a brief gripping movement of the hand. Longer trains of stimuli produced a sustained contraction. Stimuli commonly evoked synergistic responses in multiple muscles, probably due to activation of local spinal circuitry in addition to direct excitation of motoneurons. At threshold for movement, stimuli evoked responses in a single muscle at only $14 \%$ of effective sites, whereas two to six muscles were simultaneously activated at $47 \%$ of sites. Finger movements were the most commonly evoked, occurring at $59 \%$ of effective sites.

\section{DISCUSSION}

Successful creation of a neural prosthetic to aid upper limb function following injury depends on solving several technical and scientific problems. Here, we have described progress in three areas: recording stable single-unit activity from primary motor cortex during unrestrained behavior, relating this activity to muscle patterns, and evoking movements of the hand and arm by intraspinal microstimulation. Clearly, a BCI system to restore the normal range of complex motor behaviors remains a formidable challenge. Arm and hand movements are performed in a high-dimensional, multijoint space with control signals distributed over large populations of cortical neurons [5]. As the computational power of the PSoC increases, it may be possible to record multiple neural channels on one chip. Alternatively, the parallel architecture that we have implemented for simultaneous spike and EMG recording could be expanded to incorporate multiple PSoCs running as independent modules. Such a system would have greater flexibility and computational power, with expansion limited primarily by space and battery considerations. Furthermore, the finding that useful functional muscle synergies can be evoked by spinal stimulation (cf. [10]) may reduce the required dimensionality of control signals.

An unresolved issue concerns the appropriate transformation between cortical signals and stimulation parameters. Linear correlation between single cells and muscle activity typically yielded modest regression coefficients. The activity of multiple cells allows calculation of a better fit of EMG envelopes [5], [12], [13] in repetitive tasks, although generalization to an unrestricted range of movements remains untested. Thus, a major factor in successful prosthetic control is the degree to which neural activity can be appropriately modified under closed-loop conditions in which the consequences of this activity are immediately evident. Previous operant conditioning studies have shown that the requisite flexibility exists for motor cortex neurons. Given biofeedback showing the degree to which cortical cell activity met criteria for reinforcement, monkeys learned within minutes to modify cell activity in various ways in order to drive a meter arm toward reinforcement threshold [14]. Cortical cells and arm muscles that were normally co-activated could be readily dissociated within minutes [15]. Cortical firing patterns have been shown to adapt during control of BCIs [4], [5], and over longer periods of time this flexibility may well facilitate adaptation to changed motor demands, such as that which occurred with chronic cross-innervation of antagonist forelimb muscles [16]. Because the Neurochip BCI allows long-term, continuous operation, we should be able to test the degree to which monkeys can learn to appropriately control motor cortical activity that directly evokes spinal microstimulation in order to generate coordinated movements. This approach also holds clinical promise: patients could learn to compensate for impaired corticospinal connections or spinal cord injury through a Neurochip BCI allowing cortical cell activity to directly evoke or facilitate movement.

\section{ACKNOWLEDGMENT}

The authors would like to thank C. Kirby for technical assistance, J. Longnion for help developing the dual chip, Dr. F. Miles for the stimulation board, and Dr. S. Perlmutter for advice and assistance.

\section{REFERENCES}

[1] K. D. Anderson, "Targeting recovery: Priorities of the spinal cord-injured population," J. Neurotrauma, vol. 21, pp. 1371-1383, Oct. 2004.

[2] R. Porter and R. N. Lemon, Corticospinal Function and Voluntary Movement. Oxford, U.K.: Clarendon, 1993.

[3] M. D. Serruya, N. G. Hatsopoulos, L. Paninski, M. R. Fellows, and J. P. Donoghue, "Instant neural control of a movement signal," Nature, vol. 416, pp. 141-142, Mar. 2002. 
[4] D. M. Taylor, S. I. Tillery, and A. B. Schwartz, "Direct cortical control of 3D neuroprosthetic devices," Science, vol. 296, pp. 1829-1832, Jun. 2002.

[5] J. M. Carmena, M. A. Lebedev, R. E. Crist, J. E. O’Doherty, D. M. Santucci, D. F. Dimitrov, P. G. Patil, C. S. Henriquez, and M. A. Nicolelis, "Learning to control a brain-machine interface for reaching and grasping by primates," PLoS Biol., vol. 1, p. E42, Nov. 2003.

[6] C. Diorio and J. Mavoori, "Computer electronics meet animal brains," IEEE Computer, vol. 36, pp. 69-75, Jan. 2003.

[7] J. Mavoori, A. Jackson, C. Diorio, and E. E. Fetz, "An autonomous implantable computer for neural recording and stimulation in unrestrained primates," J. Neurosci. Meth., vol. 148, pp. 71-77, Oct. 2005.

[8] D. R. Humphrey, E. M. Schmidt, and W. D. Thompson, "Predicting measures of motor performance from multiple cortical spike trains," Science, vol. 170, pp. 758-762, Nov. 1970.

[9] R. N. Holdefer and L. E. Miller, "Primary motor cortical neurons encode functional muscle synergies," Exp. Brain Res., vol. 146, pp. 233-243, Sep. 2002.

[10] V. K. Mushahwar, D. F. Collins, and A. Prochazka, "Spinal cord microstimulation generates functional limb movements in chronically implanted cats," Exp. Neurol., vol. 163, pp. 422-429, Jun. 2000.

[11] S. I. Perlmutter, M. A. Maier, and E. E. Fetz, "Activity of spinal interneurons and their effects on forearm muscles during voluntary wrist movements in the monkey," J. Neurophysiol., vol. 80, pp. 2475-2494, Nov. 1998.

[12] M. M. Morrow and L. E. Miller, "Prediction of muscle activity by populations of sequentially recorded primary motor cortex neurons," J. Neurophysiol., vol. 89, pp. 2279-2288, Apr. 2003.

[13] D. T. Westwick, E. A. Pohlmeyer, S. A. Solla, L. E. Miller, and E. J. Perreault, "Identification of multiple-input systems with highly coupled inputs: application to EMG prediction from multiple intracortical electrodes," Neural Comput., vol. 18, pp. 329-355, Feb. 2006.

[14] E. E. Fetz and M. A. Baker, "Operantly conditioned patterns of precentral unit activity and correlated responses in adjacent cells and contralateral muscles," J. Neurophysiol., no. 36, pp. 179-204, Mar. 1973.

[15] E. E. Fetz and D. V. Finocchio, "Correlations between activity of motor cortex cells and arm muscles during operantly conditioned response patterns," Exp. Brain Res., vol. 23, pp. 217-240, Sep. 1975.

[16] C. Brinkman, R. Porter, and J. Norman, "Plasticity of motor behavior in monkeys with crossed forelimb nerves," Science, vol. 220, pp. 438-440, Apr. 1983.

\section{EEG and MEG Brain-Computer Interface for Tetraplegic Patients}

Laura Kauhanen, Tommi Nykopp, Janne Lehtonen, Pasi Jylänki, Jukka Heikkonen, Pekka Rantanen, Hannu Alaranta, and Mikko Sams

Abstract-We characterized features of magnetoencephalographic (MEG) and electroencephalographic (EEG) signals generated in the sensorimotor cortex of three tetraplegics attempting index finger movements. Single MEG and EEG trials were classified offline into two classes using two different classifiers, a batch trained classifier and a dynamic classifier. Classification accuracies obtained with dynamic classifier were better, at $75 \%, 89 \%$, and $91 \%$ in different subjects, when features were in the $0.5-3.0-H z$ frequency band. Classification accuracies of EEG and MEG did not differ.

Index Terms-Brain-computer interface (BCI), dynamic classification, electroencephalographic (EEG), MEG, tetraplegia.

\section{INTRODUCTION}

Brain-computer interface (BCI) research has been conducted in the Helsinki University of Technology since 1998. We were partners in an EU-funded project Adaptive Brain Interfaces from 1998 to 2001. The Academy of Finland funded the project On-line Adaptive Brain-Computer Interface from 2003 to 2005. We are a partner in an EU-funded project called Non Invasive Brain Interaction with Robots-Mental Augmentation through Determination of Intended Action. Currently, our team consists of two senior researchers, three Ph.D. students, and two undergraduate students. Our tetraplegic subjects are undergoing rehabilitation at the Käpylä Rehabilitation Centre, Helsinki, Finland.

We have developed a MATLAB-based BCI system that can be used for both offline and online research [1]. It has a graphical user interface for fast and easy handling of subject information, recordings, and model building. A program that transfers electroencephalographic (EEG) data to MATLAB in near real time has been developed. We have tested functionality of the design in an online experiment in which four healthy subjects performed cued finger extensions every $2 \mathrm{~s}$. Mean classification accuracy was $73.5 \%$ [2].

In our EEG-based BCI, artificial neural networks are used to recognize and classify brain activation patterns associated with real and attempted movements. We have also examined the use of magnetoencephalographic (MEG) signals, which are more localized than EEG signals. The 306-channel MEG device used allows simultaneous measurements of EEG. We have examined offline classification of single MEG trials of data from five subjects [3]. Classification accuracy of the left versus right finger movements was $80 \%-94 \%$, quite similar as obtained in previous comparable EEG studies.

Manuscript received July 17, 2005; revised March 20, 2006. This work was supported in part by the Academy of Finland under Projects 200849 and 49881 and by the European IST Programme FET under Project FP6-003758.

L. Kauhanen, T. Nykopp, J. Lehtonen, P. Jylänki, and J. Heikkonen are with the Laboratory of Computational Engineering, Helsinki University of Technology, FIN-02015 Espoo, Finland (e-mail: laura.kauhanen@tkk.fi).

P. Rantanen is with the ORTON Orthopaedic Hospital, Invalid Foundation, FIN-00280 Helsinki, Finland.

H. Alaranta is with the Finnish Association of People with Mobility Disabilities, Käpylä Rehabilitation Centre, FIN-00251 Helsinki, Finland.

M. Sams is with the Laboratory of Computational Engineering, Helsinki University of Technology, FIN-02015 Espoo, Finland and also with the Low Temperature Laboratory, Helsinki University of Technology, FIN-02015 Espoo, Finland.

Digital Object Identifier 10.1109/TNSRE.2006.875546 